\title{
Wavefront tracking within the stationary phase approximation
}

\author{
Johannes Bahrdt \\ BESSYGmbH, Albert-Einstein-Strasse 15, 12489 Berlin, Germany
}

(Received 1 December 2006; published 12 June 2007)

\begin{abstract}
A physical optics approach to wavefront tracking is presented based on the stationary phase approximation. It allows for modeling transmission of radiation through a system of optical elements including coherence properties with an acceptable numerical effort. The code PHASE incorporating these ideas assumes small angles as it is the case for most synchrotron radiation and the free electron laser beam lines.
\end{abstract}

PACS numbers: 42.25.- $\mathrm{p}, 07.85 . \mathrm{Q}$

\section{INTRODUCTION}

The next generation of synchrotron radiation facilities is based on free electron laser (FEL) sources. The radiation properties are superior to those of storage rings in many aspects. The peak brightness overcomes the numbers of third generation machines by 10 orders of magnitude; the pulse length is in the fs-regime; the light is transversely coherent (SASE [1-3] and HGHG [4-13]) and longitudinally coherent (HGHG).

The photon beam quality depends strongly on the parameters of the electron gun, the linac, and the FEL undulators. Extensive start-to-end simulations of the electron beam have been performed at various laboratories to estimate the radiation properties at the end of the last undulator module including fabrication and alignment tolerances.

The user of an FEL facility is interested in the beam quality at the experiment. Consequently, the start-to-end simulations have to be extended to include also the photon beam line. Ray tracing codes used in the past are generally based on geometrical optics. These codes cannot simulate directly the longitudinal and transverse coherence properties of the FEL radiation. Wavefront propagation tools have to be used instead. Furthermore, a realistic wavefront propagation is essential in cases where combined FEL and beam line simulations are required, e.g., self-seeding $[14,15]$ or spectral cleaning of the photon beam within two HGHG stages [16,17]. The complete radiation pulse as provided by time dependent FEL simulations has to be propagated in these cases. Table I shows the advantages of physical optics codes with respect to geometrical optics codes. In geometrical optics intensities rather than complex amplitudes are evaluated and, thus, phase dependent effects such as the diffraction at an aperture, the variation of the state of polarization along the beam line, or time dependent effects are principally not included. The diffraction at an aperture can artificially be implemented into a ray tracing code using an idealized parametrization of the photon beam (e.g. plane wave or spherical wave) and analytical equations for the angular distribution of the rays behind the aperture. Furthermore, the path length of the rays can be monitored which gives some information on the pulse lengthening due to an optical element.
Nevertheless, these are crude approximations which cannot describe the detailed propagation of a realistic beam and therefore we label these features as "indirect" in Table I.

A physical optics approach may also be required for the design of specific beam lines at third generation synchrotron radiation facilities. Depending on the electron beam emittance $\varepsilon$ and the betatron function $\beta$, the electron beam size and divergence, $\sigma_{\text {electron }}$ and $\sigma_{\text {electron }}^{\prime}$, can be significantly smaller than the photon beam size and divergence. In this case the radiation is transversally coherent:

$$
\begin{gathered}
\sigma_{\text {electron }}=\sqrt{\varepsilon \cdot \beta} \ll \sigma_{\text {photon }}=\frac{1}{\pi \sqrt{2}} \sqrt{\lambda \cdot L} \\
\sigma_{\text {electron }}^{\prime}=\sqrt{\varepsilon / \beta} \ll \sigma_{\text {photon }}^{\prime}=\sqrt{\lambda / 2 L} .
\end{gathered}
$$

The photon beam parameters are given for an undulator with the length $L$ and a photon wavelength of $\lambda$. The vertical electron beam emittance of third generation storage rings is smaller than $1^{-10} \pi$ mrad whereas the horizontal emittance is typically 1-2 orders of magnitude larger. Therefore, the undulator radiation is vertically coherent up to several hundred $\mathrm{eV}$ whereas it is only partially coherent or incoherent in the horizontal plane. Most beam lines have a vertical dispersion plane and the important coherent properties in this plane can be simulated with physical optics codes. If necessary, the partially coherent or inco-

TABLE I. Comparison of geometrical and physical optics

\begin{tabular}{|c|c|c|}
\hline & $\begin{array}{l}\text { Geometrical } \\
\text { optics }\end{array}$ & $\begin{array}{l}\text { Physical } \\
\text { optics }\end{array}$ \\
\hline Total intensity & Yes & Yes \\
\hline Brightness & Yes & Yes \\
\hline Field amplitudes & No & Yes \\
\hline Diffraction limited source & No & Yes \\
\hline Diffraction effects of beam line & Indirect & Yes \\
\hline $\begin{array}{l}\text { Polarization modulation within } \\
\text { the beam line }\end{array}$ & No & Yes \\
\hline Time structure & Indirect & Yes \\
\hline
\end{tabular}
codes. 
herent properties in the other plane can be included as well by averaging over different initial electron beam parameters which is, however, very time consuming.

Fourier optics techniques are generally used for the propagation of wavefronts. In this paper we present a different physical optics approach. We discuss the stationary phase approximation for synchrotron radiation and free electron laser beam lines and describe the code PHASE $[15,18,19]$ which is based on this approximation. It provides more flexibility in the choice of the grid parameters in the source and the image plane. This has advantages in systems with strong optical aberrations like coma or in cases where the complete 2-dimensional field distribution does not have to be evaluated. Furthermore, the thin lens approximation of the Fourier optics method is not needed and, finally, a sequence of optical elements can be simulated in a single step.

In Sec. II the propagation of radiation in free space and across normal incidence optics using the Fourier optics approach is briefly reviewed. In the thin lens (short mirror) approximation, this method can be extended to grazing incidence optics. Section III describes the new method of wavefront propagation for the case of a single grazing incidence optical element. In Sec. IV this method is extended to a sequence of grazing incidence elements using a matrix formalism. In Sec. V the proper choice of the integration parameters is discussed. Time dependent simulations are described in Sec. VI. In Sec. VII the implementation of the formalism into the code PHASE is presented and in Sec. VIII the new method is compared to Fourier optics propagation algorithms.

\section{FREE SPACE PROPAGATION AND NORMAL INCIDENCE OPTICS}

The electric field inside a closed volume is uniquely defined by the fields and their normal derivatives at the boundaries. Thus, the free space propagation of an electric field distribution defined on an infinitely wide screen can be obtained as an exact solution of Maxwell's equations without further approximations. With the introduction of optical elements such as apertures or mirrors, certain assumptions of the field properties on the boundaries (e.g. at the backside of an aperture) are required. These assumptions will be discussed in the following.

According to the principle of Huygens and Fresnel, the propagation of monochromatic light is completely defined by the amplitude distribution on a (curved) wavefront. Each point on the wavefront acts as a source for a spherical wave. The electric field is propagated via the propagation of the individual point sources and the results are superimposed in the image plane. An obliquity factor as introduced by Fresnel excludes the propagation in the backward direction. The factor has the value of one in the forward direction and zero in the direction perpendicular to the direction of propagation. Within the scalar diffraction the- ory, Kirchhoff solved the Helmholtz equation behind an aperture which is illuminated by a point source in front of this aperture deriving an explicit expression for the obliquity factor $K$ :

$$
\begin{aligned}
\vec{E}\left(z^{\prime}, y^{\prime}\right) & =K \cdot \int_{-\infty}^{\infty} \int_{-\infty}^{\infty} \vec{E}(z, y) \frac{e^{i k \cdot \vec{r}-\vec{r}^{\prime} \mid}}{\left|\vec{r}-\vec{r}^{\prime}\right|} \cdot d y \cdot d z \\
K & =-\frac{i}{2 \lambda}\left(\vec{n} \cdot \vec{r} / r-\vec{n} \cdot \vec{r}^{\prime} / r^{\prime}\right) .
\end{aligned}
$$

$\vec{n}$ is the normal of the surface element and $\vec{r}$ and $\vec{r}^{\prime}$ are the vectors between the surface element and a point source and the point of observation, respectively. Kirchhoff's expression is based on the assumptions that (i) the field and the normal derivative of the field vanish everywhere on the boundary except at the aperture and (ii) the field at the aperture is described by the point source. The first assumption overdetermines the boundary problem. The solution is already uniquely defined for either Dirichlet or Neumann conditions. Therefore, this assumption implies zero fields everywhere inside the boundary which is in contradiction to the second assumption. Mathematically consistent solutions of the Helmholtz equation can be obtained from approximate solutions of the fields or normal field derivatives on the boundary using either Dirichlet or Neumanntype Green's functions [20]. The corresponding obliquity factors are

$$
K_{D}=-\frac{i}{\lambda}\left(\vec{n} \cdot \vec{r}^{\prime} / r^{\prime}\right) \quad K_{N}=-\frac{i}{\lambda}(-\vec{n} \cdot \vec{r} / r) .
$$

Since the field or the field derivative on the back side of the aperture is not known, we cannot decide for one or the other obliquity factor. The divergence of synchrotron radiation or FEL photon beams in the VUV and x-ray regime is small and the distances between the optical elements and the elements and the source and image planes are usually large as compared to the optical elements. In this case the obliquity factor is constant and does not need to be evaluated explicitly.

For photon beams with a small divergence, the longitudinal electric field component can be ignored and the beam properties are well described by the transverse distribution of the electric fields in a plane perpendicular to the direction of propagation. Then, the electric fields can be propagated by a direct integration of the equation of Fresnel and Kirchhoff [Eq. (1)] which is, however, slow.

Alternatively, in Fourier optics [21,22] various methods are used depending on whether the image is in the near field or the far field.

In the near field case, the original field distribution is usually decomposed into plane waves with different orientations using a Fourier transformation. The drift $\Delta x$ is applied by multiplication of an appropriate phase factor. A Fourier back transformation yields the field distribution at the new location: 


$$
\begin{gathered}
\vec{E}_{0}\left(\nu_{z}, \nu_{y}\right)=\int_{-\infty}^{\infty} \int_{-\infty}^{\infty} \vec{E}(z, y) \cdot e^{-2 \pi i\left(\nu_{z} z+\nu_{y} y\right)} d y \cdot d z \\
\vec{E}\left(\nu_{z}, \nu_{y}\right)=\vec{E}_{0}\left(\nu_{z}, \nu_{y}\right) \cdot e^{2 \pi i \cdot \Delta x \cdot \sqrt{1 / \lambda^{2}-\nu_{z}^{2}-\nu_{y}^{2}}} \\
\vec{E}\left(z^{\prime}, y^{\prime}\right)=\int_{-\infty}^{\infty} \int_{-\infty}^{\infty} \vec{E}\left(\nu_{z}, \nu_{y}\right) \cdot e^{2 \pi i \cdot\left(\nu_{z} z+\nu_{y} y\right)} \cdot d \nu_{y} \cdot d \nu_{z} .
\end{gathered}
$$

In the far field case, the original field distribution is convoluted with the so-called point spread function which describes the propagation of an individual point source. The convolution integral follows directly from a Fourier transformation of Eq. (3). Rewriting the expression yields

$$
\begin{aligned}
\vec{E}\left(y^{\prime}, z^{\prime}, \Delta x\right)= & \frac{1}{i \lambda \Delta x} e^{i\left[\left(k r^{\prime 2}\right) /(2 \Delta x)\right]} \\
& \times \mathrm{FFT}^{ \pm}\left[\vec{E}(y, z, 0) \cdot e^{i\left[\left(k r^{2}\right) /(2 \Delta x)\right]}\right] .
\end{aligned}
$$

FFT indicates a fast Fourier transformation and the sign of the Fourier transformation depends on the direction of propagation. The variables of the Fourier transform are $y^{\prime} / \lambda z$ and $z^{\prime} / \lambda z$.

Both methods are mathematically equivalent and no approximations have been applied so far. Depending on the Fresnel number of the geometry, one or the other method may be more appropriate to minimize the numerical noise introduced by the phase factors which comprise the propagation distance $\Delta x$ in the nominator and the denominator, respectively.

For large propagation distances the phase factors of Eq. (5) are nearly constant and can be dropped (Fraunhofer approximation). Then, the propagation is described by

$$
\vec{E}\left(y^{\prime}, z^{\prime}, \Delta x\right)=\frac{1}{i \lambda \Delta x} \mathrm{FFT}^{ \pm}[\vec{E}(y, z, 0)] .
$$

Depending on the location of the source and the image plane with respect to the beam waist and depending on the Rayleigh range, an appropriate combination of the propagators above can be chosen for any free space propagation. For non-Gaussian beams we define the beam waist as the

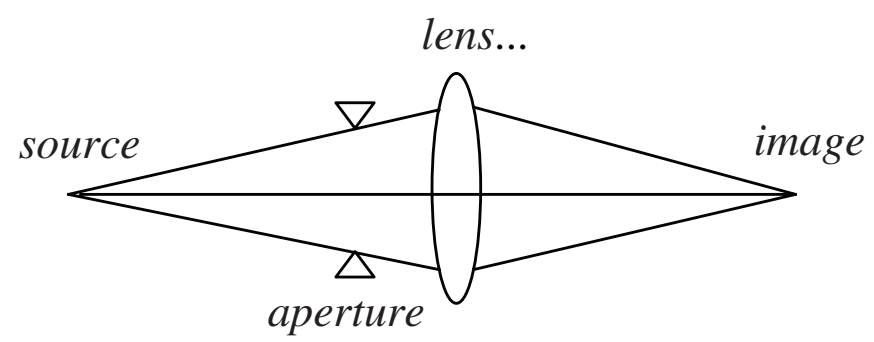

FIG. 1. Layout of a normal incidence geometry. longitudinal position with the smallest transverse beam dimension in terms of the standard deviation of a fitted Gaussian function. The waist location in the horizontal and vertical direction may be different.

The propagation across a normal incidence element such as a lens, zone plate, aperture, etc. (Fig. 1) is done in three steps: (i) free space propagation to the center of the element using one of the three propagators defined in Eq. (1), Eqs. (2)-(4), or Eq. (5) or a combination of them; (ii) multiplication of the electric fields with an appropriate complex matrix; (iii) propagation of the field to the image plane.

\section{GRAZING INCIDENCE OPTICS}

Grazing incidence geometries can be simulated in Fourier optics using the following procedure (e.g. GLAD [23] or ZEMAX [24] Manual): (i) free space propagation of the electric field to the optical element; (ii) ray tracing across the optical element including the phase advance; (iii) free space propagation to the next element.

The second step ignores diffraction effects along the optical element which is justified for thin lenses or short mirrors.

In the following, a different method is introduced which is based on the stationary phase approximation [25]. It is partially described in $[15,18,19]$ and forms the basis of the physical optics computer code PHASE. We consider a geometry with two planes (source and image plane) normal to the direction of propagation and located on either side of an optical element. A monochromatic beam of radiation with wave vector $k$ and wavelength $\lambda$ propagates from the source plane to the image plane being reflected or diffracted at a mirror or grating, respectively (Fig. 2).

The electric field distribution $\vec{E}\left(\vec{a}^{\prime}\right)$ in the image plane due to the field distribution $\vec{E}(\vec{a})$ is written using the propagator $h$ [26]:

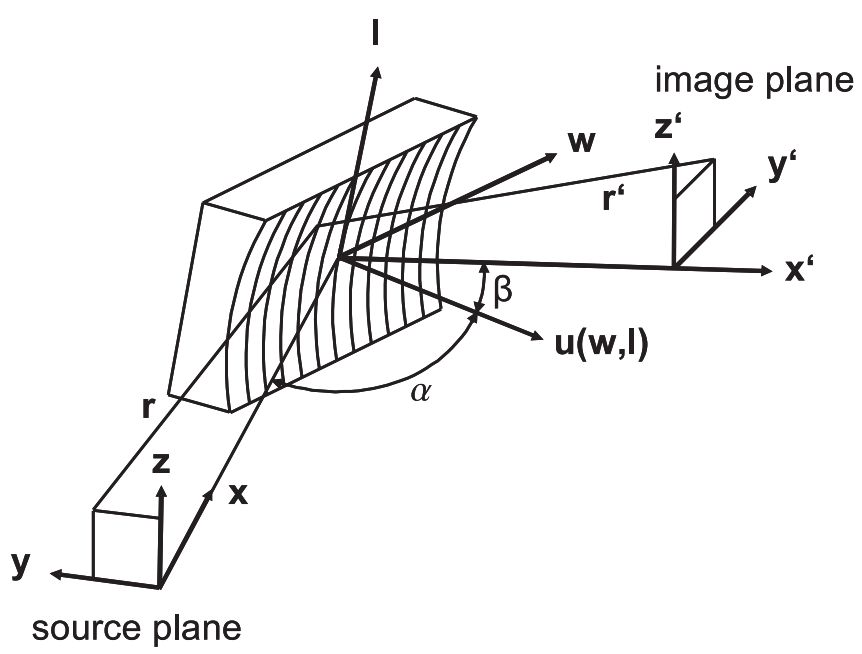

FIG. 2. Coordinate system and definition of variables. 


$$
\begin{aligned}
\vec{E}\left(\vec{a}^{\prime}\right) & =\int h\left(\vec{a}^{\prime}, \vec{a}\right) \cdot \vec{E}(\vec{a}) \cdot d \vec{a} \\
h\left(\vec{a}^{\prime}, \vec{a}\right) & =K \int_{\text {surface }} \frac{\exp \left(i k\left(r+r^{\prime}\right)\right)}{r r^{\prime}} \cdot b(w, l) \cdot d w \cdot d l,
\end{aligned}
$$

where $b$ is the transmittance of the optical element, $K$ the obliquity factor which is assumed to be constant, and $r$ and $r^{\prime}$ are the distances from the source to the optical element and from the optical element to the image plane, respectively.

The integrations in Eq. (7) are performed over the source plane and the active area of the optical element. Both the propagator $h$ and the transmittance $b$ of the optical element are here taken to be scalar quantities excluding polarizing properties of the optical element from the discussion. Polarization effects are easily included by writing $\vec{E}$ in terms of two orthogonal polarization vectors and replacing $b$ by the corresponding Jones matrix [27]. Then $h$ becomes a $2 \times 2$ matrix, too.

The obliquity factor $K$ varies only little over the optical element surface. Knowing of the mathematical inconsistence we approximate it by

$$
K=-\frac{i}{2 \lambda}[\cos (\alpha)+\cos (\beta)],
$$

where $\alpha$ and $\beta$ are the angles between the normal of the optical element and the ingoing and outgoing central ray, respectively. For a mirror we have $\alpha=\beta$.

For typical grazing incidence optics at synchrotron radiation light sources the propagator of one optical element can be simplified applying the stationary phase approximation [25]. This approximation is based on the fact that the asymptotic behavior $(k \rightarrow \infty)$ of the integral of the propagator $h$ in Eq. (7) is completely defined by the behavior of the integrand at the critical points. The critical points of the first kind are locations $\left(w_{0}, l_{0}\right)$ where the first derivatives of the path length $\mathrm{PL}=r+r^{\prime}$ with respect to $w$ and $l$ are zero (the second derivatives are nonzero) and the critical points of the second kind are the points of stationary phase on the boundaries. If the optical element does not scrape the beam, the dominant term in the asymptotic expansion is the contribution from the critical points of the first kind. In geometrical optics these points are the intersections of the rays with the optical element surface.

Expanding the optical path length (PL) in Eq. (7) around the point $\left(w_{0}, l_{0}\right)$ we obtain

$$
\begin{aligned}
h\left(\vec{a}, \vec{a}^{\prime}\right) \propto & \int \frac{1}{r r^{\prime}} \exp \left[i k\left(\sum_{m, n} \frac{\partial^{m+n} \mathrm{PL}}{\partial \Delta w^{m} \partial \Delta l^{n}} \cdot \frac{\Delta w^{m} \cdot \Delta l^{n}}{m ! \cdot n !}\right)\right] \\
& \cdot d \Delta w \cdot d \Delta l \\
& w=w_{0}+\Delta w \quad l=l_{0}+\Delta l .
\end{aligned}
$$

We approximate $r$ and $r^{\prime}$ by the distances to the critical points $r_{w_{0} l_{0}}$ and $r^{\prime}{ }_{w_{0}, l_{0}}$, respectively. The terms linear in $\Delta w$ and $\Delta l$ vanish by definition of the critical point. The leading term of the contributions from the critical points of the first kind is the term containing the second order expansion of the optical path length:

$$
\begin{aligned}
h\left(\vec{a}, \vec{a}^{\prime}\right) \propto & \frac{1}{r_{w_{0}, l_{0}} \cdot r^{\prime}{ }_{w_{0}, l_{0}}} \exp \left[i k\left(r_{w_{0}, l_{0}}+r_{w_{0}, l_{0}}^{\prime}\right)\right] \\
& \cdot \int \exp \left[i k \left(\frac{\partial^{2} \mathrm{PL}}{\partial \Delta w^{2}} \cdot \frac{\Delta w^{2}}{2}+\frac{\partial^{2} \mathrm{PL}}{\partial \Delta l^{2}} \cdot \frac{\Delta l^{2}}{2}\right.\right. \\
& \left.\left.+\frac{\partial^{2} \mathrm{PL}}{\partial \Delta w \cdot \partial \Delta l} \Delta w \cdot \Delta l\right)\right] \cdot d \Delta w \cdot d \Delta l .
\end{aligned}
$$

The coordinate system of the optical element can be rotated such that the cross term in Eq. (9) vanishes and the integral can be separated into two individual integrals [25]. The two integrals can be solved analytically if the integration limits are set to infinity. This is justified if the critical points of the first kind are well separated (see Sec. V) and the mirrors are sufficiently large. Apart from a path length dependent term each integral contains a factor of $e^{ \pm i \pi / 4}$ where the sign depends on the sign of the second derivatives of the path length. For an aperture or a plane mirror, obviously this factor cancels the imaginary prefactor of the obliquity factor.

With this simplification Eq. (7) reduces to Eq. (10) which is written in the nonrotated coordinate system. The dimensions of integration have been reduced from 4 to 2 :

$$
\begin{aligned}
\vec{E}\left(\vec{a}^{\prime}\right) \propto & \int \vec{E}(\vec{a}) \cdot \exp \left[i k\left(r_{w_{0}, l_{0}}+r_{w_{0}, l_{0}}^{\prime}\right)\right] /\left(r_{w_{0}, l_{0}} \cdot r_{w_{0}, l_{0}}^{\prime}\right) \\
& \cdot\left|\frac{\partial^{2} \mathrm{PL}}{\partial \Delta w^{2}} \cdot \frac{\partial^{2} \mathrm{PL}}{\partial \Delta l^{2}}-\left(\frac{\partial^{2} \mathrm{PL}}{\partial \Delta w \cdot \partial \Delta l}\right)^{2}\right|^{-1 / 2} \cdot d \vec{a} .
\end{aligned}
$$

In [25] an analytic expression for the leading term of the contributions from the critical points of the second kind is given. This expression is, however, not regarded here because we assume that in most cases the optical elements do not scrape the photon beam. In cases where this assumption is not true the propagation can be split into two steps where the beam is propagated from the source to an aperture in front of the limiting element and, then, propagated to the image plane.

For typical synchrotron radiation beam lines (small divergencies, distances between optical elements much larger than sizes of optical elements), it can be shown numerically that for mirrors or gratings the following relation holds with an accuracy better than $10^{-3}$ :

$$
\begin{aligned}
& \left|\frac{\partial^{2} \mathrm{PL}}{\partial \Delta w^{2}} \cdot \frac{\partial^{2} \mathrm{PL}}{\partial \Delta l^{2}}-\left(\frac{\partial^{2} \mathrm{PL}}{\partial \Delta w \cdot \partial \Delta l}\right)^{2}\right| \\
& \cong \frac{\cos (\alpha) \cdot \cos (\beta)}{r^{2} r^{\prime 2}} \cdot\left|\frac{\partial(y, z)}{\partial\left(d y^{\prime}, d z^{\prime}\right)}\right| .
\end{aligned}
$$

Note that the derivatives are taken at the point where the principle ray hits the element. Once, the coordinate/angle transformation over a sequence of optical elements is 
known, the right-hand side of Eq. (11) can easily be evaluated for the combination of elements. An analytical derivation of the limits of Eq. (11) for a single optical element still has to be done. Furthermore, the accuracy for a combination of optical elements has to be determined. In the next chapter, we present a general description of a sequence of elements which does not make use of Eq. (11).

\section{SEQUENCE OF OPTICAL ELEMENTS}

In this chapter, we will present a general approach to propagate a wavefront across set of $N$ optical elements. The method is similar to the one described in the last section for one optical element.

For a combination of several optical elements it is advantageous to substitute the source coordinates $[\vec{a}=$ $(z, y)]$ with the divergences in the image plane:

$$
\vec{E}\left(\vec{a}^{\prime}\right)=\int h\left(\vec{a}^{\prime}, \vec{a}\right) \cdot \vec{E}(\vec{a}) \cdot\left|\frac{\partial(y, z)}{\partial\left(d y^{\prime}, d z^{\prime}\right)}\right| \cdot d\left(d y^{\prime}\right) \cdot d\left(d z^{\prime}\right)
$$

using the functional determinant $|\cdots|$. Similarly, the optical element coordinates $w, l$ are expanded in terms of the image coordinates and angles. The relations $y\left(d y^{\prime}, d z^{\prime}\right)$ and $z\left(d y^{\prime}, d z^{\prime}\right)$ are obtained from geometrical optics.

The propagator for two optical elements is given by an integral over the product of two individual propagators of the two elements:

$$
h\left(\vec{a}^{\prime \prime}, \vec{a}\right)=\int h_{2}\left(\vec{a}^{\prime \prime}, \vec{a}^{\prime}\right) \cdot h_{1}\left(\vec{a}^{\prime}, \vec{a}\right) \cdot d \vec{a}^{\prime} .
$$

Equation (13) represents a six-dimensional integral where four dimensions correspond to the two surface integrations and two dimensions are due to the integration across the intermediate plane. Rewriting the integral, the latter integration can be skipped. Thus, the dimensionality of the integral increases by two with each additional element and an approximation is required to keep the computation time below reasonable values.

Before we start, we briefly review the generation of the transformation map across $N$ optical elements. Based on geometrical optics, a fourth order Taylor series expansion of the final coordinates $\left(y_{f}, z_{f}, d y_{f}, d z_{f}\right)$ with respect to the initial coordinates $\left(y_{i}, z_{i}, d y_{i}, d z_{i}\right)$ is evaluated. In this context the final and initial coordinates are the source and image coordinates, respectively. Using these expressions, the expansion coefficients of all cross products up to fourth order are generated. The transformation of all products can be represented by a $70 \times 70$ transformation matrix. In this way, the generally nonlinear transformation of the coordinates and angles can be expressed by a linear operation:

$$
\bar{Y}_{f}=\overline{\bar{M}} \cdot \bar{Y}_{i} \quad \bar{Y}_{f}=\left(y_{f}, z_{f}, d y_{f}, d z_{f}, y_{f}^{2}, y_{f} z_{f} \ldots\right)
$$

and similar for the initial coordinates $\bar{Y}_{i}$.

The transformation across several optical elements is simply described by the product matrix of all individual matrices:

$$
\overline{Y_{m}}=\left(\prod_{k=m+1}^{N} \overline{\overline{M_{k}}}\right) \cdot \overline{Y_{N}}
$$

where $\bar{Y}_{N}$ are the initial and $\bar{Y}_{m}$ the final coordinates.

The amplitude contribution of a specific source point to a specific image point is given by the sum over all possible paths [28] (Fig. 3).

There are three types of contributions to the optical path length. They are described by different analytical expressions:

$$
\begin{aligned}
\mathrm{PL}_{1} & =f_{1}\left(y, z, w_{1}, l_{1}\right) \\
\mathrm{PL}_{i+1} & =f_{2}\left(w_{i}, l_{i}, w_{i+1}, l_{i+1}\right) \\
\mathrm{PL}_{N+1} & =f_{N+1}\left(w_{N}, l_{N}, y^{\prime}, z^{\prime}\right) \\
\mathrm{PL} & =\mathrm{PL}_{1}+\sum_{i=2}^{N} \mathrm{PL}_{i}+\mathrm{PL}_{N+1} .
\end{aligned}
$$

We substitute the optical element coordinates with

$$
w_{i}=w_{i 0}+\Delta w_{i} \quad l_{i}=l_{i 0}+\Delta l_{i}
$$

and expand the optical path PL with respect to the small displacements relative to the critical points of the first kind at all optical elements $\left\{\left(w_{10}, l_{10}\right) \ldots\left(w_{N 0}, l_{N 0}\right)\right\}$ and with respect to the image coordinates. For $N=1$ we have

$$
\begin{aligned}
\mathrm{PL}= & \sum_{k l m n r s} \operatorname{PLC}(k, l, m, n, r, s) \cdot y_{i}^{k} \cdot z_{i}^{l} \cdot d y_{i}^{m} \cdot d z_{i}^{n} \\
& \cdot \Delta w^{r} \cdot \Delta l^{s},
\end{aligned}
$$

where PLC are the expansion coefficients of the path

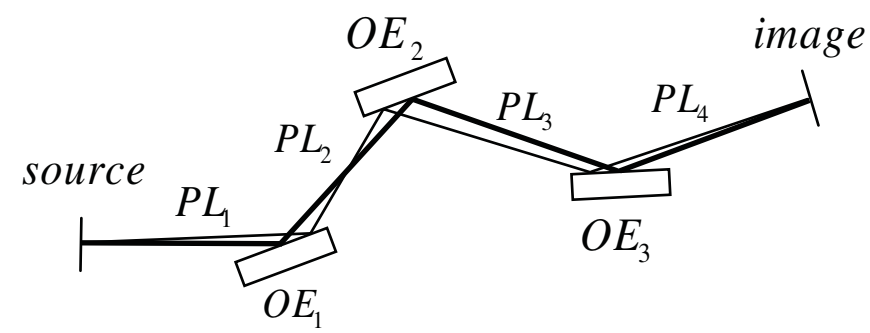

FIG. 3. The principle ray (thick line) between a source and an image point has zero derivatives with respect to all optical element coordinates. In geometrical optics, only these rays are regarded. In physical optics, all possible paths have to be evaluated (e.g. thin line). 
length. As an example, we get for a single plane mirror

$\operatorname{PLC}(0,0,0,0,2,0)=\frac{1}{2 r_{0}}+\frac{1}{2 r_{0}^{\prime}} \quad \operatorname{PLC}(0,0,0,0,4,0)=-\frac{1}{8 r_{0}^{3}}-\frac{1}{8 r_{0}{ }^{\prime 3}} \quad r_{0}^{(\prime)}=r^{(\prime)}{ }_{w_{0}} l_{0} \quad\left(w_{0}=0, l_{0}=0\right)$.

For $N$ optical elements, the path length has the form

$$
\begin{aligned}
& \mathrm{PL}=\quad \sum_{p, q=1 \ldots N} \operatorname{PLC}\left(k, l, m, n, r_{p}, s_{q}\right) \cdot y_{i}^{k} \cdot z_{i}^{l} \cdot d y_{i}^{m} \cdot d z_{i}^{n} \cdot \Delta w_{p}^{r_{p}} \cdot \Delta l_{q}^{s_{q}} . \\
& r_{p}=\{0,2\} \\
& s_{q}=\{0,2\} \\
& r_{p}+s_{q}=2 \\
& k+l+m+n \leq 4
\end{aligned}
$$

We have terminated the expansion with respect to the mirror coordinates to second order. First order terms do not appear since we expand around the ray of stationary phase. The following complex integral has to be evaluated to account for all possible optical paths where the $2 N$-tuple $\left(w_{10}, l_{10}, \ldots w_{N 0}, l_{N 0}\right)$ characterizes the ray of the stationary phase:

$$
\begin{aligned}
\tilde{h}\left(w_{10}, l_{10}, \ldots w_{N 0}, l_{N 0}\right) \propto & \frac{1}{\prod_{i=1}^{N+1} r_{0}^{i}} \cdot \exp \left[i k\left(r_{0}^{1}+\cdots+r_{0}^{N+1}\right)\right] \\
& \cdot \int \cdots \int \exp \left[i k\left(\sum_{p, q=1}^{N} \sum_{r_{p}+s_{q}=2}^{2} \frac{\partial^{r_{p}+s_{q}} \mathrm{PL}}{\partial \Delta w_{p}^{r_{p}} \cdot \partial \Delta l_{q}^{s_{q}}} \cdot \frac{\Delta w_{p}^{r_{p}} \cdot \Delta l_{q}^{s_{q}}}{r_{p} ! \cdot s_{q} !}\right)\right] \cdot d \Delta w_{1} \cdot d \Delta l_{1} \cdots d \Delta l_{N} .
\end{aligned}
$$

The tilde of the propagator indicates an integration over a sequence of optical elements. For the limiting case of one element it corresponds to the propagator of Eq. (9). The quantities $r_{0}^{1}, \ldots, r_{0}^{N+1}$ are the segment lengths of the principle ray between succeeding elements including the source and image plane.

The path length PL in the exponent can be interpreted as a $2 \mathrm{~N}$-dimensional quadratic form which can be expressed as

$$
\begin{aligned}
\mathrm{PL} & =\vec{X}^{T} \cdot \overline{\bar{G}} \cdot \vec{X} \\
\vec{X} & =\left(x_{1}, \ldots x_{2 N}\right)=\left(\Delta w_{1}, \Delta l_{1} \ldots \Delta l_{N}\right) .
\end{aligned}
$$

The matrix $\overline{\bar{G}}$ is real and symmetric. It contains all expansion coefficients PLC and all cross products of the initial coordinates and angles. The vector $\vec{X}$ represents all variables $\Delta w_{i}, \Delta l_{j}$. For each real and symmetric $N$-dimensional matrix an orthogonal transformation $\overline{\bar{K}}$ exists which diagonalizes the matrix. The diagonal elements are the eigenvalues

$$
\left(\begin{array}{ccc}
\lambda_{1} & 0 & 0 \\
0 & \cdots & 0 \\
0 & 0 & \lambda_{N}
\end{array}\right)=\overline{\bar{K}}^{T} \cdot \overline{\bar{G}} \cdot \overline{\bar{K}}
$$

We change the coordinates of the quadratic form PL using the orthogonal transformation $\overline{\bar{K}}$ :

$$
\vec{X}=\overline{\bar{K}} \cdot \vec{V} \quad \vec{V}=\left(v_{1} \ldots v_{2 N}\right)
$$

In the new coordinate system the cross terms drop out (principal axis theorem) and the quadratic form transforms to

$$
\mathrm{PL}=\sum_{i, j} g_{i j} \cdot x_{i} x_{j}=\sum_{i} \lambda_{i} \cdot v_{i}^{2}
$$

Using the functional determinant of the transformation $\overline{\bar{K}}$, the differential of integral Eq. (21) is replaced by

$$
\begin{gathered}
d \Delta w_{1} \cdot d \Delta l_{1} \cdots d \Delta l_{N}=\frac{\partial\left(\Delta w_{1} \cdots \Delta l_{N}\right)}{\partial\left(v_{1} \cdots v_{2 N}\right)} d v_{1} \\
\cdot d v_{2} \cdots d v_{2 N} .
\end{gathered}
$$

The value of the functional determinant is \pm 1 (plus sign for a pure $2 N$ dimensional rigid rotation). In the new coordinate system, the integral of Eq. (21) separates into individual integrals which can be integrated analytically when the integration limits are set to infinity: 


$$
\begin{aligned}
\tilde{h}\left(w_{10}, l_{10}, \ldots w_{N 0}, l_{N 0}\right) \propto & \frac{1}{\prod_{i=1}^{N+1} r_{0}^{i}} \cdot \frac{\partial\left(\Delta w_{1} \cdots \Delta l_{N}\right)}{\partial\left(v_{1} \cdots v_{2 N}\right)} \cdot \exp \left[i k\left(r_{0}^{1}+\cdots r_{0}^{N+1}\right)\right] \\
& \cdot \int \exp \left[i k\left(\lambda_{1} v_{1}^{2}\right)\right] d v_{1} \cdots \int \exp \left[i k\left(\lambda_{2 N} v_{2 N}^{2}\right)\right] d v_{2 N} \\
= & \frac{1}{\prod_{i=1}^{N+1} r_{i}} \cdot \frac{\partial\left(\Delta w_{1} \cdots \Delta l_{N}\right)}{\partial\left(v_{1} \cdots v_{2 N}\right)} \cdot\left(\frac{2 \pi}{k}\right)^{N} \cdot \frac{1}{\sqrt{\left|\lambda_{1} \cdots \lambda_{2 N}\right|}} \cdot e^{i m \pi / 2} \cdot e^{-i N \pi / 2} .
\end{aligned}
$$

$m$ is the number of positive eigenvalues. The determinant describing the quadratic form is invariant for orthogonal transformation and we can replace

$$
\lambda_{1} \cdots \lambda_{2 N}=|\overline{\bar{G}}| .
$$

For two variables (one optical element, $N=1$ ), $m$ can be evaluated from the two invariants trace and determinant [25]. This is not possible for more than two variables. We only know that for a positive or negative determinant $m$ is even or odd. In the latter case we get an additional factor of $i$. The sign ambiguity in the evaluation of the integral cannot be resolved. The sign can be different for different quadruples $\left(y_{i}, z_{i}, d y_{i}, d z_{i}\right)$. The sign of the integral is, however, constant as long as the signs of the eigenvalues are constant. It is unreasonable that the signs change under smooth variations of the initial coordinates unless one eigenvalue has a zero crossing. The zero crossing corresponds to a zero second derivative of the path length and a zero determinant. For these cases the algorithm cannot be applied (see Sec. V). The integral can be evaluated by extrapolating the data from adjacent quadruples $\left(y_{i}, z_{i}, d y_{i}, d z_{i}\right)$.

In principle, the sign can be determined by solving the eigenvalue problem. For matrices of order $4 \times 4$ or less symbolic solutions (containing only addition, subtraction, multiplication, division, and roots of the matrix elements) can always be found starting from the characteristic polynomial. For higher orders this is not always possible (AbelRuffini theorem). Generally, iterative methods are used in those cases like the Jacobi method or the Householder method with subsequent solution of the characteristic polynomial with Newton's procedure [29]. In our case the matrix elements are 4th order power series expansions with respect to the initial coordinates and the eigenvalue problem cannot be solved symbolically. On the other hand, a numerical solution for each quadruple $\left(y_{i}, z_{i}, d y_{i}, d z_{i}\right)$ would be rather time consuming. Therefore, we do not solve the eigenvalue problem at all but evaluate only the determinant of the original representation matrix $\overline{\bar{G}}$ and stay with the sign uncertainty. This is acceptable since in the end we are interested in intensities. The determinant itself can be evaluated analytically.

The matrix $\overline{\bar{G}}$ is symmetric and it is sparse since combined partial derivatives of non-neighboring optical elements are zero:

$$
\frac{\partial^{2} \mathrm{PL}}{\partial \Delta w_{i} \partial \Delta l_{j}}=0, \forall i, j:\{i<j-1 \vee i>j+1\} .
$$

A system of three optical elements has the following non zero matrix elements:

$$
\overline{\bar{G}}=\left[\begin{array}{cccccc}
g_{w_{1} w_{1}} & g_{w_{1} l_{1}} & g_{w_{1} w_{2}} & g_{w_{2} l_{2}} & 0 & 0 \\
g_{l_{1} w_{1}} & g_{l_{1} l_{1}} & g_{l_{1} w_{2}} & g_{l_{1} l_{2}} & 0 & 0 \\
g_{w_{2} w_{1}} & g_{w_{2} l_{1}} & g_{w_{2} w_{2}} & g_{w_{2} l_{2}} & g_{w_{2} w_{3}} & g_{w_{2} l_{3}} \\
g_{l_{2} w_{1}} & g_{l_{2} l_{1}} & g_{l_{2} w_{2}} & g_{l_{2} l_{2}} & g_{l_{2} w_{3}} & g_{l_{2} l_{3}} \\
0 & 0 & g_{w_{3} w_{2}} & g_{w_{3} l_{2}} & g_{w_{3} w_{3}} & g_{w_{3} l_{3}} \\
0 & 0 & g_{l_{3} w_{2}} & g_{l_{3} l_{2}} & g_{l_{3} w_{3}} & g_{l_{3} l_{3}}
\end{array}\right] .
$$

Each matrix element is a fourth order polynomial of the variables $\left(y_{i}, z_{i}, d y_{i}, d z_{i}\right)$. Using these expressions, the determinant of $\overline{\bar{G}}$ can be expressed as a fourth order expansion of the same variables. The evaluation of the expansion coefficients of the expressions in Eq. (27) is fast as compared to the wavefront propagation and it has to be done only once for a given geometry.

\section{SINGULARITIES}

The approximations above are based on the following assumptions: (i) The optical elements do not scrape the beam. (ii) The second derivative of the path length is always finite in order to avoid singularities. This implies that the source and the image plane are not simultaneously focal planes (neither in horizontal nor in vertical direction). (iii) The critical points of the first kind are well separated.

The last assumption would be dispensable if the optical path length is expanded up to third order in the optical element coordinates. Then, the integration could be performed even close to the singularities. There is, however, no solution to separate the integral of Eq. (21) in this case which is essential for an analytic integration.

In the following we will discuss the validity of the third assumption.

\section{A. Single optical element}

First, we consider a strongly focusing single optical element under grazing incidence conditions. In this case a specific optical aberration named coma shows up. It describes the effect that two different principle rays connect identical points in the source and the image plane. Depending on the parameters these two rays can be well 
separated or can strongly interfere. The interference may happen also in cases where the source and image planes are not simultaneously focal planes.

We consider the following geometry: a toroidal mirror operates at $2^{\circ}$ grazing incidence and demagnifies by a factor 20:1; the distances between the mirror and the focal planes are 10 and $0.5 \mathrm{~m}$; the electric field is given $5.0 \mathrm{~m}$ upstream of the mirror.

The argument of the integral in Eq. (21) contains cosand sin-functions of the optical path length. In Fig. 4 the path lengths between two points in the source and image plane and its cosine are plotted versus the intersection point at the optical element. Three cases are identified: Two principal rays which are well separated show up [Fig. 4(a)]; two principal rays interfere [Fig. 4(b)]; there are no stationary rays at all but the contribution to the integral cannot be neglected [Fig. 4(c)]. The three examples differ only in the vertical coordinates of the start and end points in the source and the image plane.

In the stationary phase approximation, the third and higher order path length expansion coefficients are neglected. If the second order derivations approach zero, the inverse of the determinant of Eq. (28) diverges. Even for zero second derivatives the contribution to the result may still be finite if the functional determinant of Eq. (12) is also zero. The error, however, may be large because third order terms get important and will also contribute to the result (second and third case).

The specific behavior of the rays of the stationary phase depends strongly on the geometry parameters. For different distances between the source plane and the first mirror the location of the principal rays varies. This provides a reliable indication on the validity of the approximations: (i) the determinant must be nonzero for all cases (this can easily be checked during propagation); (ii) the result must be insensitive to the choice of the distance between the source plane and the first optical element.

One can choose the location of the source plane such that one of the principal rays is shifted well beyond the aperture of the optical element and no interference effects occur. For the given geometry, the results are stable for source-mirror distances smaller than approximately $1.5 \mathrm{~m}$ (Fig. 5). For larger distances, interference effects get important. The curves do not diverge in the regions of small second derivatives of the path length because the functional determinant in Eq. (12) has small values at the same time. Nevertheless, the interference effects deteriorate the results (Fig. 5). The appropriate choice of the location of the source plane does not imply a loss of generality since the corresponding electric field distribution can always be propagated to any location before starting the propagation across the beam line.

In the cases of only one or two optical elements, it may be possible to elaborate a criterion for the validity of assumption (iii): In a first step the cross terms of the optical
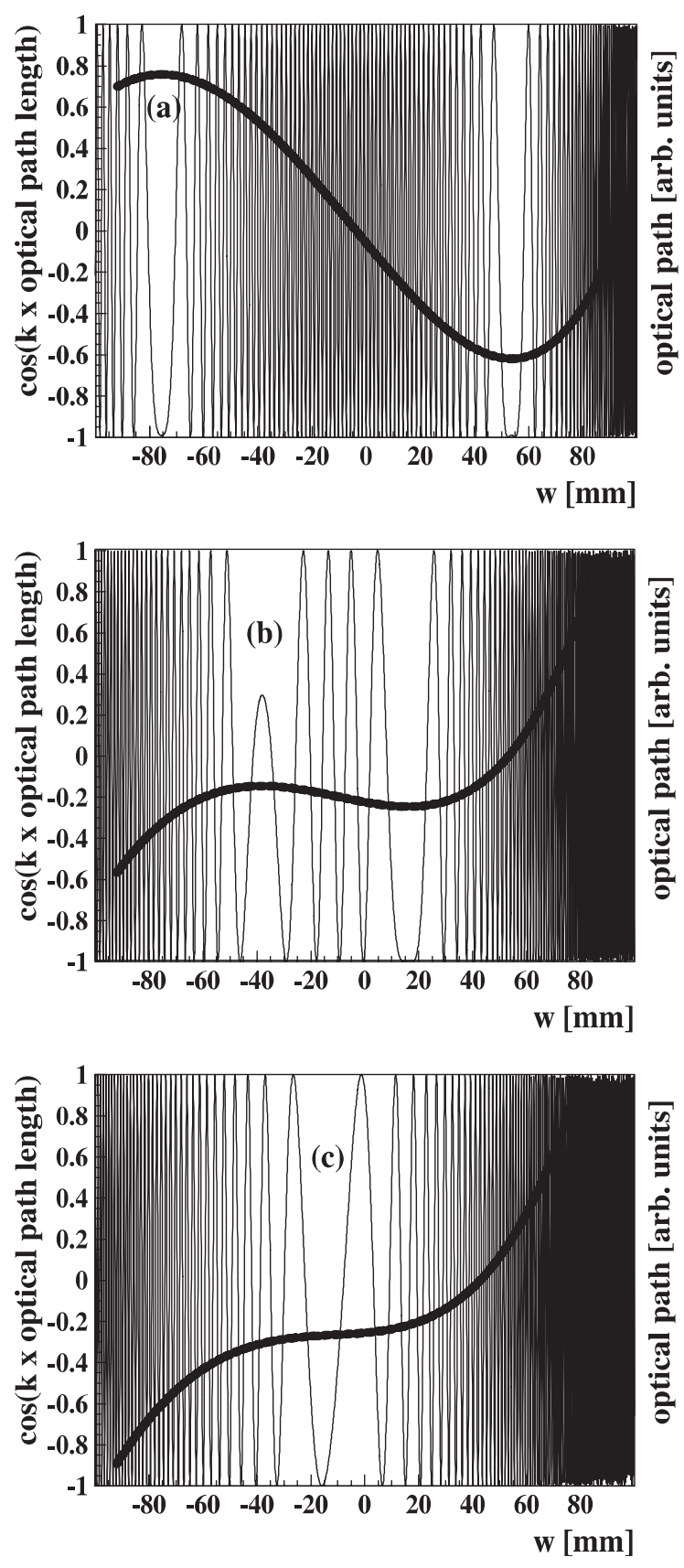

FIG. 4. (a) Paths of stationary phase are well separated. (b) Paths of stationary phase interfere. (c) There is no path of stationary phase but the contribution to the integral cannot be neglected.

path length expansion are removed via an analytical principle axis transformation. Then, an integration interval for the transformed surface coordinates has to be determined such that the integration over this interval delivers results close to the values of an integration up to infinity. If the third order terms within this interval are small as compared to the second order terms, the assumption is justified. This topic will be subject of further studies in the future. 


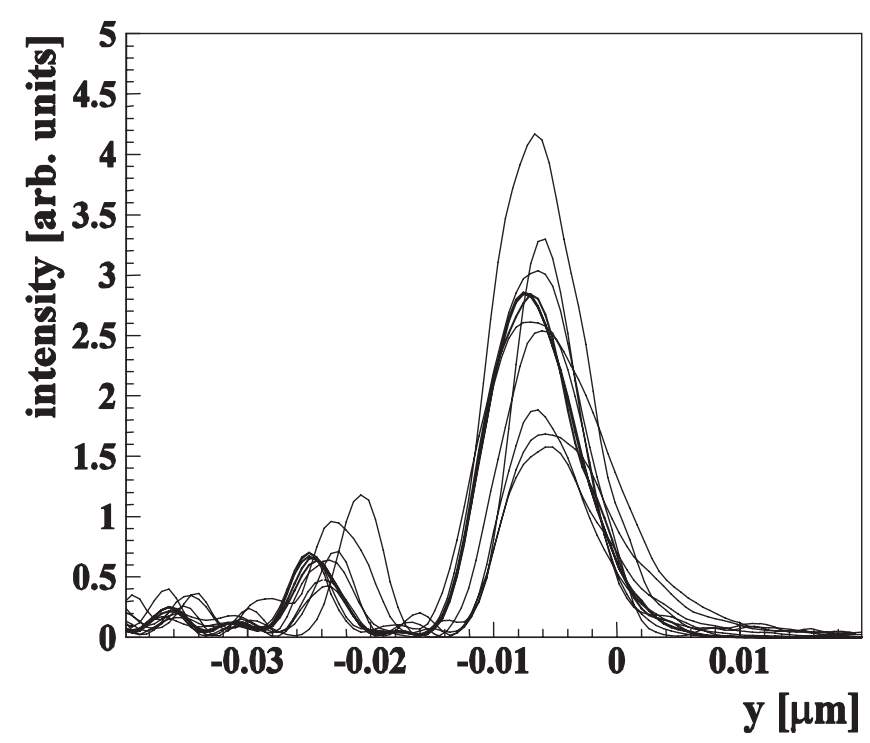

FIG. 5. Vertical cut in the focal plane for the geometry described in the text. The thick lines which are nearly identical correspond to source-mirror distances of $0.5,1.0$, and $1.5 \mathrm{~m}$, respectively. The thin lines are the results for distances of 2.0, 3.0, 4.0, 5.0, 6.0, 7.0, 8.0, and $9.0 \mathrm{~m}$.

\section{B. Sequence of optical elements}

As demonstrated in Sec. VA for a single optical element, the existence of several critical points for fixed coordinates $(y, z)$ and $\left(y^{\prime}, z^{\prime}\right)$ can spoil the result if they are not well separated. For a sequence of optical elements two cases have to be distinguished: The two partial derivatives of the optical path length are performed: (i) at one optical ele-
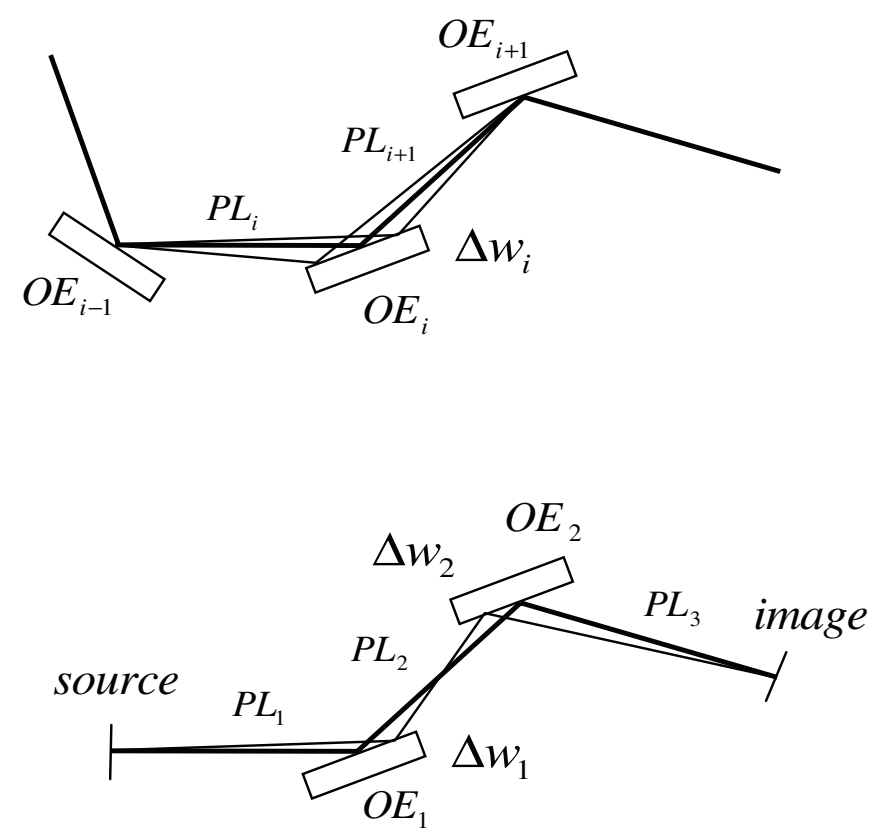

FIG. 6. Top: Variation of one coordinate of one optical element $\mathrm{OE}_{i}$. Bottom: Variation of the coordinates of two neighboring optical elements. ment with respect to one coordinate or (ii) at one or more elements with respect to different coordinates. In the first case (Fig. 6, top), the angle of incidence at the next, the previous or both neighboring optical elements changes when the variables $\Delta w_{i}$ or $\Delta l_{i}$ are varied. Since the other rays are kept fixed the law of reflection which describes the rays of stationary phase does not apply. Hence, within the region of integration there exists only one principle ray. In the second case, the variables can be either coordinates of the same optical element $\left(\Delta w_{i}\right.$ and $\left.\Delta l_{i}\right)$ or neighboring elements (Fig. 6, bottom). An arrangement of two optical elements may have several principle rays for various combinations of $\Delta w_{1}$ and $\Delta w_{2}$. The source plane has to be chosen appropriately to exclude these cases (see Sec. VA). For three or more optical elements the existence of only one principle ray can again be concluded from the law of reflection.

\section{TIME DEPENDENT SIMULATIONS}

The fractional frequency bandwidth of the new soft $x$ ray and $\mathrm{x}$-ray FELs is typically a few 0.001 . To take this into account the simulations have to be performed for various frequencies. The time dependent FEL code GENESIS [30] provides the complex electric field vectors on a transverse grid. In the time dependent mode, a large number of slices represents the time evolution of the electric fields at each grid point. Before starting the electric field propagation the time dependent electric fields $\vec{E}(t)$ have to be converted to frequency dependent variables $\vec{E}(\zeta)$ via a Fourier transformation for each grid point. For a pure drift, one of the algorithms described in Eqs. (2)(4), Eq. (5), or Eq. (6) is applied to the transverse field distributions of each frequency. If optical elements are included, the procedures described in Sec. III and IV have to be used. Finally, the resulting frequency slices are back transformed to time space.

GENESIS evaluates photon beam properties at the end of the FEL where the radiation is already divergent. Following the described procedure, these data can be propagated upstream in order to derive information on the effective location of the photon beam waist and the size of the phase space [31]. These data which may depend on the frequency are required for a proper beam line design.

The short time structure of an HGHG FEL radiation pulse can be spoiled within a beam line. Optical aberrations can cause path length differences. The effects are, however, of minor importance for low divergent FEL beams. The influence of a grating can be significant. In geometrical optics the path length difference $\Delta L(\Delta y)$ at a transmission grating (Fig. 7) causes a pulse lengthening of

$$
\Delta t=\frac{n \lambda}{\cos (\alpha) \cdot c} \Delta y
$$

where $n$ is the line density, $c$ is the velocity of light, and $\Delta y$ 

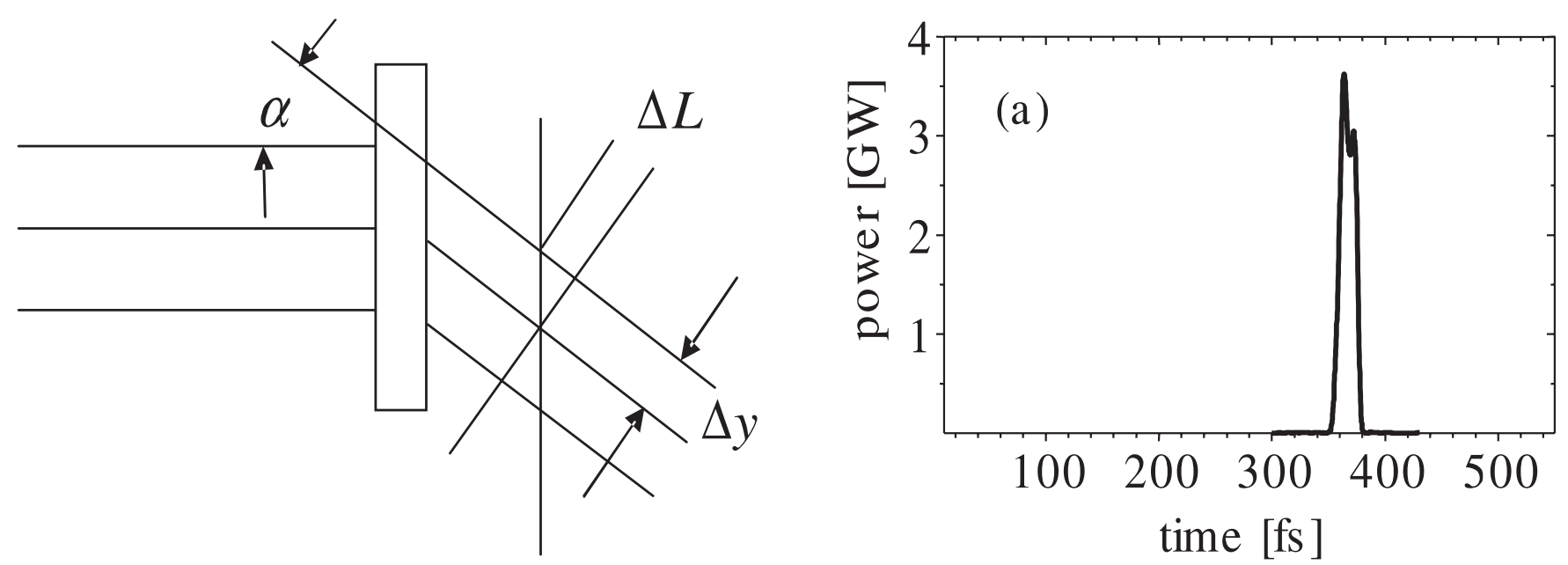

FIG. 7. Pulse lengthening at a transmission grating.

characterizes the displacement in the dispersion direction. In physical optics the transverse field distribution experiences an additional phase variation of $\Delta \Phi=f(\Delta y)$ :

$$
\vec{E}(\zeta, \Delta y)=\vec{E}_{0}(\zeta, \Delta y) \cdot e^{i \cdot \Delta \Phi} \quad \Delta \Phi=2 \pi \zeta \cdot \Delta t,
$$

where $\zeta$ is the frequency and $\Delta t$ is defined in Eq. (31).

The Fourier transformation of this product is the convolution of the time dependent field distribution with a $\delta$-function which is equivalent to the result in geometrical optics:

$$
\vec{E}(t, \Delta y)=\operatorname{FFT}^{-1}[\vec{E}(\zeta, \Delta y)]=\vec{E}_{0}(t, \Delta y) \otimes \delta(t-\Delta t) .
$$

Monochromatic waves are infinitely long. To simulate time dependent effects, the simulations have to be extended to a set of frequencies. Assuming only minor phase variations within the FEL pulse the radiation can be approximated by

$$
\overrightarrow{\hat{E}}(z, y, t)=\overrightarrow{\vec{E}}_{0}(z, y) \cdot g(t) .
$$

The electric field distribution $\vec{E}_{0}(z, y)$ is extracted from a time independent FEL simulation and $g(t)$ is a real, slowly varying (on the scale of an optical period) function which simulates the time structure. This approximation might be justified for idealized HGHG radiation which is longitudinally fully coherent.

For detailed simulations, however, a set of electric field distributions at various locations within the electron bunch has to be used. The number of slices depends on the time structure and the phase variation within the bunch. Obviously, many more slices are needed for a SASE case as compared to a HGHG case [see Figs. 8(a) and 8(b)].

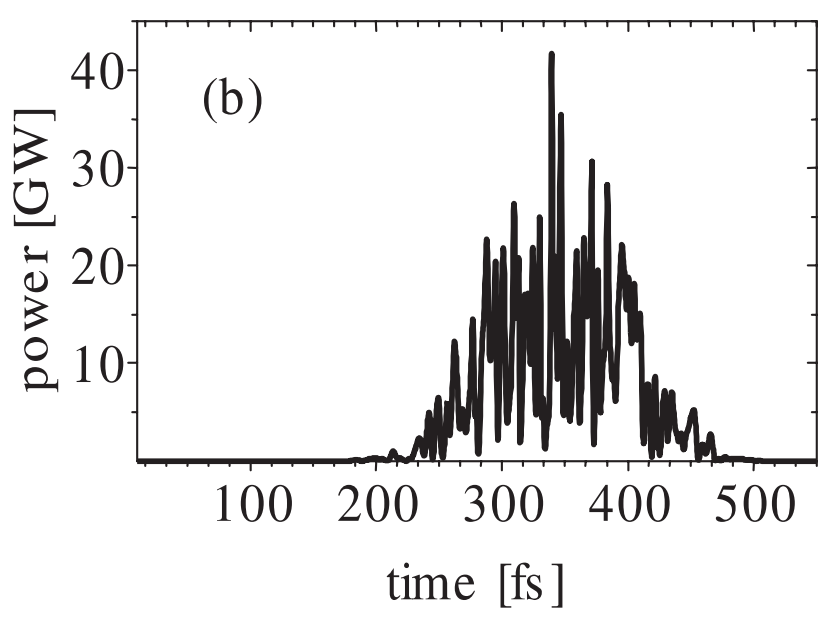

FIG. 8. (a) Typical time structure of an HGHG-FEL. (b) Typical time structure of a SASE-FEL.

\section{THE PHYSICAL OPTICS CODE PHASE}

Most of the equations discussed above have been implemented into the code PHASE. The scaling factor Eq. (27) for a combination of several optical elements is not yet included in the code and Eq. (11) is used instead. The implementation of Eq. (27) will be done in the future.

The representation of the source is a key issue in order to keep the CPU time low. A typical electric field distribution (real and imaginary part) for the spontaneous radiation of an undulator is given in Fig. 9, top. The fast oscillating quantities require a fine mesh for integration. The fields can be converted to amplitudes and phases. The discontinuities of the phase distribution can be removed by an appropriate addition of multiples of $2 \pi$ (Fig. 9, bottom). Furthermore, the quadratic phase dependency which might be different in both planes can be subtracted as well. Then, the mesh can be coarse which significantly reduces the CPU time.

The optical element surfaces are described by 5 th order polynomials. All Taylor series expansions have been performed up to fourth order in the image coordinates 



FIG. 9. Electric field distribution of a spontaneous undulator source (top) and an equivalent amplitude and phase representation of the same source (bottom).

$\left(y^{\prime}, z^{\prime}, d y^{\prime}, d z^{\prime}\right)$ which is adequate for most geometries. The accuracy might not be sufficient for optical elements with a strong curvature perpendicular to the optical axis (strong demagnifications) where higher order terms are needed. In principle, higher order Taylor series expansions can be implemented. This affects mainly the size of the code which is less important for modern computers (e.g. increase of a factor of 10 going from third and fourth order).
On the other hand, for higher expansion orders the integration time increases by less than a factor of 2 going from one to the next order of Taylor series expansion (Table II). The evaluation of the transfer maps and scaling factors is fast and has to be done only once for a given geometry.

After the geometries and optical elements have been defined, the parameters are checked and optimized in the fast running ray tracing mode (geometrical optic). Slope 
TABLE II. Number of expansion coefficients to be evaluated during integration for various expansion orders.

\begin{tabular}{cc}
\hline \hline Expansion order & Expansion coefficients \\
\hline 2 & 15 \\
3 & 35 \\
4 & 70 \\
5 & 126 \\
6 & 210 \\
\hline \hline
\end{tabular}

errors as well as misalignments of the optical elements can be taken into account in this mode. The code provides the possibility to automatically minimize any linear combination of matrix elements of the transformation map (optical aberrations) by variation of defined geometry or mirror parameters [32]. Once the beam line parameters are fixed, one switches to the physical optics mode and performs the propagation for the defined parameter set.

Even with optimized integration parameters, time dependent simulations typically take hours on a single CPU machine. For the propagation of SASE FEL radiation where many frequencies have to be included, the program should be implemented on a multi-CPU cluster. The code is suitable for being parallelized since each frequency is independently simulated.

The code is written partly in FORTRAN and partly in C. The power series expansions have all been evaluated using the algebraic code REDUCE [33] which automatically generates FORTRAN code. The graphics are realized with the PAW package of the CERN library. The mouse driven user interface is based on the Motif library. The code runs on a LINUX platform. Currently, PHASE is a monolithic program. In the future it will be rewritten as a shared library to be used in a script language. This will simplify the addition of new features.

\section{COMPARISON OF PHASE AND FOURIER OPTICS}

In the following we will discuss the aspects flexibility, speed and storage requirements.

Fourier optics techniques are based on fast Fourier transformations in two dimensions. Let us assume that the electric field is defined on a $M \times M$ grid with $M=2^{n}$. Then, the CPU time for the FFT scales with

$$
T_{\mathrm{CPU}}^{\mathrm{FFT}} \propto(n-1) \cdot M^{2} .
$$

The stationary phase approximation requires a four dimensional integration and scales like

$$
T_{\mathrm{CPU}}^{\mathrm{SPA}} \propto M^{4} .
$$

In Fig. 10 the CPU time for different wavefront propagation algorithms are compared where the Fourier optics (FO) data have been evaluated using a self-sorting version of a decimation-in-frequency procedure [34]. Times have

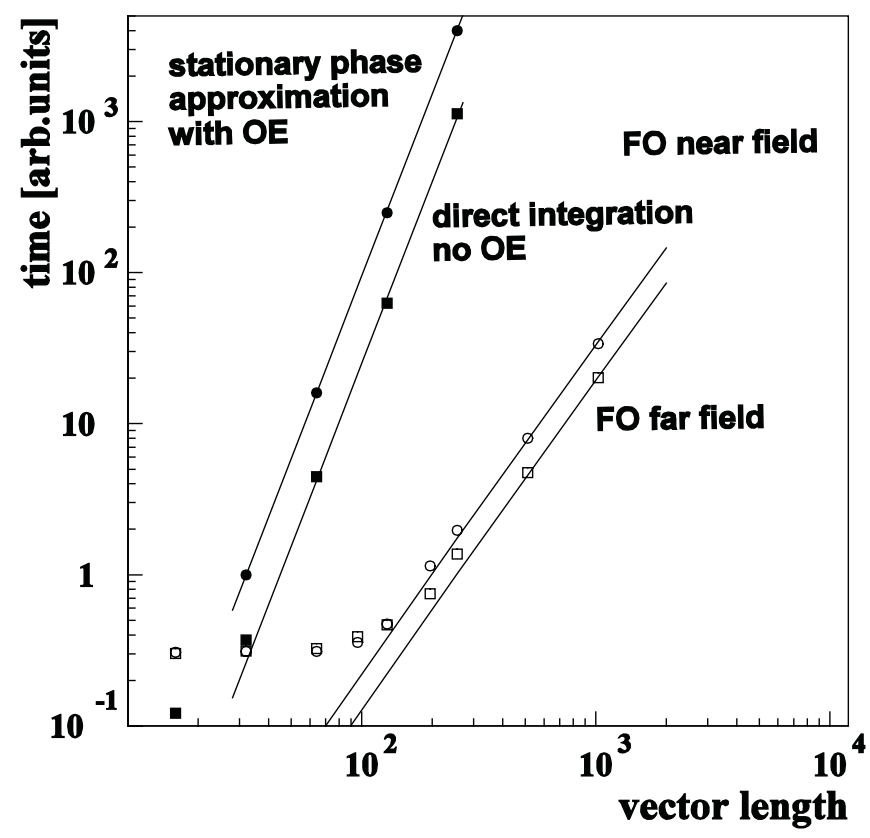

FIG. 10. The CPU time as measured for three free space propagators (Fourier optics propagation: open square and open circle, direct integration of the Fresnel Kirchhoff equation: solid square) and propagation across an optical element using the stationary phase approximation (solid circle). The lines indicate the general scaling behavior as described in the text.

been given for a single propagation. In Fourier optics at least two propagations are required for one optical element and even more for several elements. Following the PHASE algorithm, several elements can be evaluated in one step. Nevertheless, the algorithm based on the stationary phase approximation is significantly slower as compared to FFT techniques for the same size of the field array.

In Fourier optics the near field propagator (Sec. II) is used if the beam size does not vary significantly between the source and the image plane. The grid remains constant and can be well adapted to the problem. If the far field operator (Sec. II) has to be used, the mesh sizes in the source and image plane cannot be chosen independently but are related via

$$
\Delta x_{f}=\frac{\lambda \cdot \Delta z}{n_{x} \cdot \Delta x_{i}}
$$

where $n_{x} \Delta x_{i / f}$ are the horizontal extensions of the electric field in the source/image plane (and similar in vertical direction) and $\Delta z$ is the propagation distance. The resolution at the waist and in the far field is comparable if

$$
D_{x_{i}}=n_{x} \cdot \Delta x_{i}=\omega_{0} \cdot \sqrt{n_{x}} \bar{\pi},
$$

where $\omega_{0}$ is the waist in the source plane. Obviously, only the central part of the field array carries significant information and the rest of the array is filled with zeros in order to keep the resolution constant during propagation. For $N=1024$ we have 


$$
D_{x_{i}}=\sqrt{2} \sigma_{\text {field }} \sqrt{1024} \cdot \bar{\pi}=20 \cdot\left(4 \sigma_{\text {field }}\right) .
$$

This means that the array size has to be a factor of 20 larger than the central part of $4 \sigma_{\text {field }}$ where the information is concentrated.

Within the stationary phase approximation, the array sizes can be adapted to the central part and, hence, the same information can be propagated using much smaller array sizes (factor of 20 in this specific case). The sampling resolution of the radiation cone increases with $\sqrt{M}$ for Fourier optics and with $M$ for the stationary phase approximation and the ratio (resolution/CPU time) is nearly independent of $M$ for both methods. In this sense the speed of both methods is comparable. This statement is limited to nearly coherent beams.

The PHASE algorithm provides the full flexibility over the grid parameters in the source and the image plane with the following options: (i) vertical cut in the image plane (one dimensional simulation for optimization purposes); (ii) horizontal band to simulate a monochromator slit; (iii) nonequidistant grid.

It gains at least a factor of 10 in speed if the same geometry is simulated several times for different electric field distributions because most of the expressions have to be evaluated only once. Two cases have to be distinguished: (i) The beam line includes a grating: The accelerated propagation method can be applied if the frequency is constant. This is the case if many propagations for different source data sets are required to improve the statistics. (ii) The beam line does not include a grating: Also time or frequency dependent simulations profit from the speed enhancement.

As already mentioned, the field arrays to be propagated can be much smaller for the new method as compared to Fourier optics schemes. This may be advantageous for time dependent simulations where thousands of frequencies have to be propagated. If the beam is confined to a narrow region at the center, one may think of appropriate preprocessors and postprocessors which expand and reduce the data sets before and after Fourier optics propagation. Then, the information to be stored is essentially the same in both methods.

In grazing incidence systems with strong demagnifications, an optical aberration called coma shows up. The intensity in the image plane is distributed over a large area and a data reduction is not possible anymore. In Fourier optics, it has to be avoided that the intensity distribution is scraped by the image boundaries because otherwise aliasing effects may deteriorate the result (Figs. 11 and 12). In this case the Fourier optics propagation requires the evaluation of large arrays though it might be that the information is required only within a small area (e.g. a beam line aperture or the sample size). Here, the new method is more effective since the simulations can be confined to the region of interest.
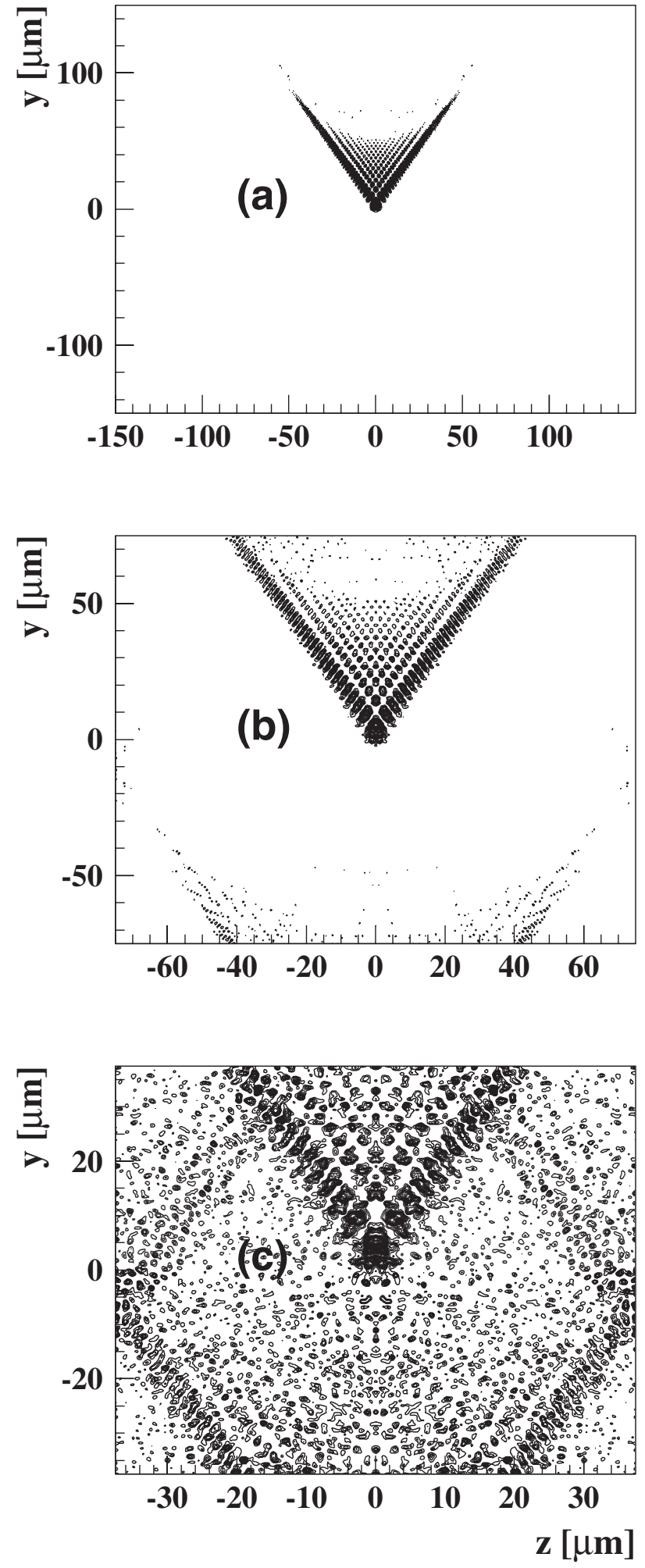

FIG. 11. Contour plots of the intensity distribution in the image plane as simulated with Fourier optics. The demagnification is 20:1, the source and image distances are 10000 and $500 \mathrm{~mm}$, and the grazing angle is $10^{\circ}$. Parts (a), (b), and (c) differ only in the image size. Numerical errors show up if the size has been chosen too small which is due to the periodic behavior of the Fourier transformation. 

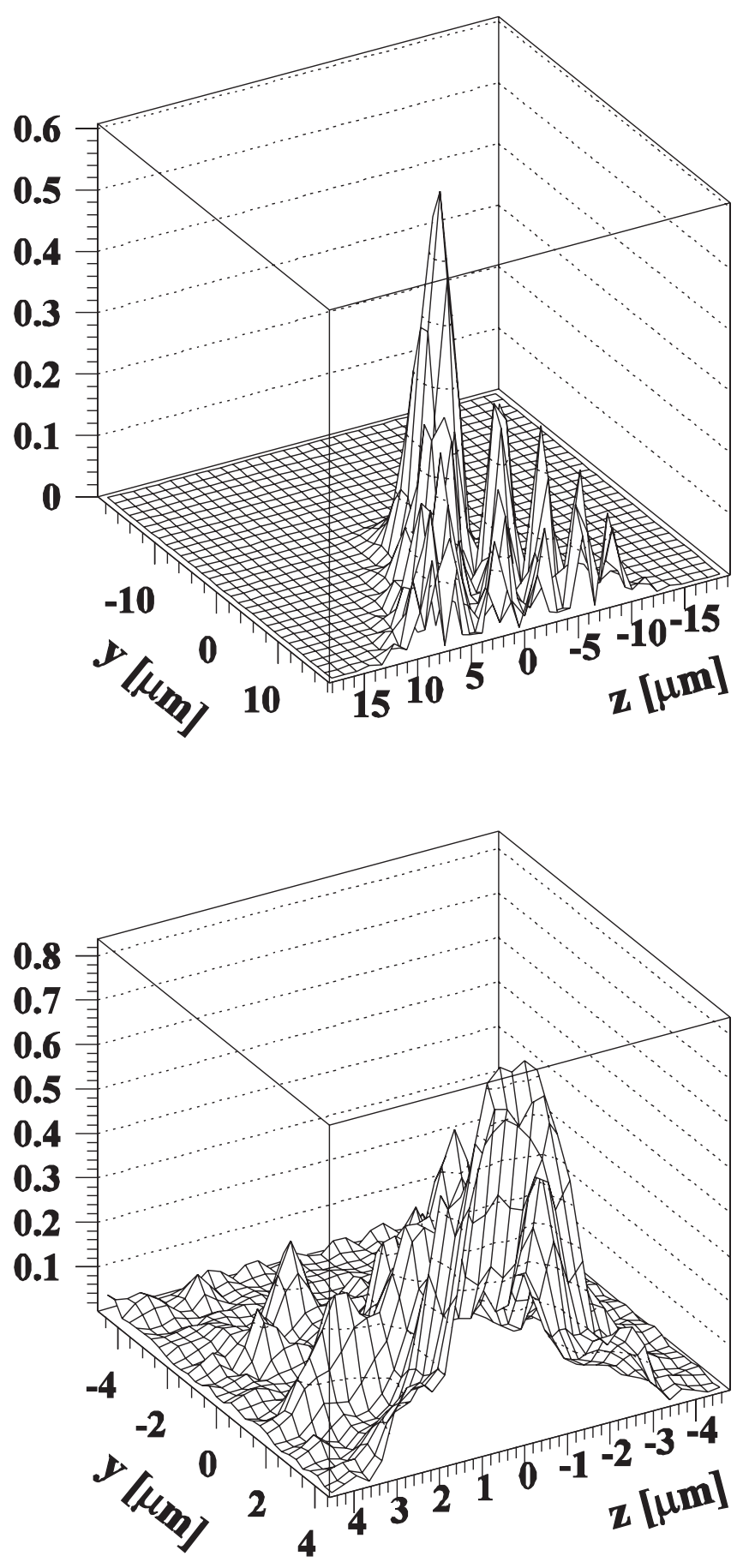

FIG. 12. Surface plots of the graphs of Fig. 11(a) (top) and Fig. 11(c) (bottom). Even at the center of the image plane, the numerical noise increases drastically if the image size is too small (bottom).

\section{CONCLUSION}

We presented a physical optics approach based on the stationary phase approximation which is well suited to propagate time dependent electric field distributions of free electron lasers. Realistic transverse and longitudinal pulse characteristics can be simulated. The algorithm has no limitations concerning the choice of the grid parameters as is the case for Fourier optics. This allows for efficient simulations comparable to the Fourier optics approach though the CPU time on the same grid is significantly longer. The storage requirements for the new propagation method are smaller which is of advantage for time/frequency dependent simulations. In Fourier optics the fields have to vanish close to the boundaries of the grid in order to avoid aliasing effects. Strongly aberrated systems with a strong coma require huge array sizes which can be simulated much more efficient with the new method.

\section{ACKNOWLEDGMENTS}

The author would like to thank A. Gaupp and G. Wüstefeld for many fruitful discussions.

[1] TESLA-Technical Design Report (Supplement), TESLAFEL Note 2002-9, edited by R. Brinkmann et al., 2002.

[2] Linac Coherent Light Source (LCLS), Conceptual Design Report, SLAC-R-593, 2002.

[3] T. Shintake, Nucl. Instrum. Methods Phys. Res., Sect. A 507, 382 (2003).

[4] R. Bonifacio et al., Nucl. Instrum. Methods Phys. Res., Sect. A 296, 787 (1990).

[5] L. H. Yu, Phys. Rev. A 44, 5178 (1991).

[6] X.J. Wang et al., Proceedings of the 26th International FEL Conference, Trieste, Italy, 2004, pp. 209-211.

[7] J. Bahrdt et al., Proceedings of the EPAC 2006, Edinburgh, Scotland, pp. 59-61.

[8] C. Bochetta et al., Proceedings of the 27th International FEL Conference, Stanford, CA, 2005, pp. 632-685.

[9] The BESSY Soft X-ray Free Electron Laser, Technical Design Report, 2004, edited by D. Krämer, E. Jaeschke, and W. Eberhardt (BESSY, Berlin, 2004).

[10] M.-E. Couprie et al., Proceedings of the 27th International FEL Conference, Stanford, CA, 2005, pp. 55-58.

[11] A. Andersson et al., Proceedings of the 26th International FEL Conference, Trieste, Italy, 2004, pp. 190-192.

[12] D. Wang et al., Proceedings of 2005 PAC, Knoxville, Tennessee, pp. 1961-1963.

[13] J. Qi-ka et al., Proceedings of the 26th International FEL Conference, Trieste, Italy, 2004, pp. 494-497.

[14] J. Feldhaus et al., Opt. Commun. 140, 341 (1997).

[15] J. Bahrdt et al., Proceedings of the 28th International FEL Conference, Berlin, Germany, 2006, pp. 521-528.

[16] K. Goldammer et al., Proceedings of the 27th International FEL Conference, Stanford, CA, 2005, pp. 23-26.

[17] M. Abo-Bakr et al., Proceedings of the 27th International FEL Conference, Stanford, CA, 2005, pp. 19-22.

[18] J. Bahrdt, Appl. Opt. 34, 114 (1995).

[19] J. Bahrdt, Appl. Opt. 36, 4367 (1997).

[20] J. D. Jackson, Classical Electrodynamics (John Wiley and Sons, New York, 1975), 2nd ed., p. 431.

[21] J. W. Goodman, Introduction to Fourier Optics, McGrawHill Physical and Quantum Electronics Series (McGrawHill, New York, 1968). 
[22] G. N. Lawrence, Optical Modeling, in Applied Optics and Optical Engineering, edited by R. R. Shannon and J.C. Wyant (Academic, New York, 1992), Vol. 11.

[23] GLAD, Theory Manual, Applied Optics Research, 1087 Lewis River Road, 217 Woodland, WA, 2004.

[24] ZEMAX, Optical Design Program, User's Manual, ZEMAX Development Corporation, 2005.

[25] L. Mandel and E. Wolf, Optical Coherence and Quantum Optics (Cambridge University Press, Cambridge, England, 1995).

[26] M. Born and E. Wolf, Principles of Optics (Pergamon Press, Oxford, 1980), 6th ed.

[27] W. B. Westerfeld et al., Appl. Opt. 24, 2256 (1985).

[28] R. Feynman, QED-The Strange Theory of Light and Matter (Princeton University Press, Princeton, 1985).

[29] W. Press, B. Flannery, S. Teukolsky, and W. Vetterling,
Numerical Recipes in C (Cambridge University Press, Cambridge, England, 1991).

[30] S. Reiche, Nucl. Instrum. Methods Phys. Res., Sect. A 429, 243 (1999).

[31] A. Meseck et al., 27th International FEL Conference, Stanford, CA, 2005, pp. 43-46.

[32] J. Bahrdt and U. Flechsig, in Gratings and Grating Monochromators for Synchrotyron Radiation, Proc. SPIE Int. Soc. Opt. Eng. Vol. 3150 (SPIE-International Society for Optical Engineering, San Diego, 1997), pp. 158-170.

[33] A. C. Hearn, REDUCE 3.5, A General Purpose Algebra System (RAND, Santa Monica, California, 1993), http:// www.uni-koeln.de/REDUCE/.

[34] PORT 3 Mathematical Subroutine Library, AT\&T Bell Laboratories, New Jersey, USA, 1984. 